\title{
Blockchain-Based Transcripts for Mobile Higher-Education
}

\author{
Timothy Arndt and Angela Guercio
}

\begin{abstract}
Blockchain has emerged as a transformative technology, from its beginning as the basis of cryptocurrencies to wider applications in areas such as property registration and insurance due to its characteristic as a distributed ledger which can remove the need for a trusted third party to facilitate transaction. This spread of the technology to new application areas has been driven by the development of smart contracts blockchain-based protocols which can automatically enforce a contract. One area where the types of problems being considered for blockchain exists is higher education. Students in higher education are increasingly mobile, and in an ever more agile world, the friction and delays caused by multiple levels of administration in higher education can cause many anxieties and hardships for students. Distance learning as a primary platform for higher education promises to open up higher education to a wider range of learners than ever before. In this paper, we review the use of blockchain in higher education and experimental implementations of a blockchain-based university transcript system in order to empower students and better fit today's ever more agile society and then describe some experimental results in using blockchain for higher education transcripts.
\end{abstract}

Index Terms-Blockchain, higher education, certification.

\section{INTRODUCTION}

Blockchain was introduced in a paper published by the pseudonymous Satoshi Nakamoto in 2008 [1] and subsequently deployed in the cryptocurrency bitcoin in the following year. Blockchain is an open, distributed ledger that can efficiently record transactions between two parties in a verifiable and immutable (permanent) fashion without the need for a trusted third party (disintermediation).

Bitcoin uses a peer-to-peer architecture and relies on proof-of-work, a piece of data which is difficult (time consuming, computationally) to produce, but easy for others to verify (via "miners" who are rewarded with bitcoin for this work) and which satisfies certain requirements as a consensus mechanism. Consensus mechanisms allow for the correctness or "truth" of a transaction to be confirmed (depending on a set of rules) when multiple distributed actors may perform transactions, and some of those actors may be untrustworthy. Blockchain thus leads to what have been called Distributed Autonomous Organisations (DAOs).

Subsequent developments have allowed blockchain to be programmed (via smart contracts) to trigger transactions automatically [2]. As a foundational technology, blockchain

Manuscript received October 10, 2019; revised December 20, 2019.

Timothy Arndt is with the Department of Information Systems, Cleveland State University, Cleveland, USA (e-mail: t.arndt@csuohio.edu).

Angela Guercio is with the Department of Computer Science, Kent State University at Stark, North Canton, USA (e-mail: aguercio@kent.edu). has been used or proposed for use in applications far beyond cryptocurrencies [3] including banking [4], land registration - especially in developing countries [5], insurance [6], and online voting [7]. Alternative consensus mechanisms (such as proof of stake and mechanisms based on Byzantine Fault Tolerance) have been proposed and alternative architectures used (e.g. client-server). These variations may be more useful in specific applications of blockchain. Some of these variations and blockchain platforms using them are discussed in the following section.

In this paper, we give an overview of some recent research on the application of blockchain in the area of higher education and describe our experimental results on implementation of a system for another promising area for blockchain technology - higher education records (university transcripts). For further study on blockchain in higher education, [8] and [9] (related works section) will be helpful. The best way to incorporate the topic of blockchain into the higher education curriculum for various academic disciplines (computer science, information systems, finance, business, etc.) is another interesting question.

\section{DISCUSSION}

In today's world, learners are more mobile than ever before. University students move from one university to another, both within one country as well as between countries. This may happen within a single degree program (student starts at a community college, moves to 4-year college $\mathrm{X}$ and then transfers to university $\mathrm{Y}$ where she completes her degree), or when completing one program and moving on to another (student completes his undergraduate degree at institute $\mathrm{X}$ in country $\mathrm{Y}$ and then starts a graduate program at institute $\mathrm{W}$ in country Z). Students today expect to be able to do this with a minimum of difficulty (for instance, credits for courses already taken should be transferred to the new institution so those courses don't have to be repeated). Blockchain technology can be used to ease these processes. In this paper, we will present some experimental systems we have developed using blockchain technology to support student mobility.

More information on our approach can be found in a previous paper [3]. In the following section, we give a deeper introduction to blockchain for higher education.

\section{BLOCKCHAIN OVERVIEW}

\section{A. Types of Blockchain}

There are currently three generally recognized types of 
blockchain systems which differ in governance and architecture [10]:

- Public blockchain - all records are visible to the public and everyone can take part in the consensus process. Immutability is high in this type of blockchain, but efficiency is low;

- Private blockchain - belong to a specific organization, and only nodes coming from that organization are allowed to join the consensus process. Compared to public blockchains, private blockchains have lower immutability, but higher efficiency;

- Consortium blockchain - a combination of the two previous types of system in which a pre-selected group of users can participate in the consensus process, and not all users belong to the same organization. Immutability and efficiency are similar to the private blockchain, while consortium blockchain is intermediate in terms of centralization between the decentralized public blockchain and centralized private blockchain. This type is also known as a permissioned blockchain.

\section{B. When Is Blockchain Useful?}

Blockchain is a highly hyped, perhaps overhyped, technology. It is not applicable to all projects. Relational databases are a more stable, longer established technology which meet the needs of many, if not most, projects. On the other hand, there are certain usage scenarios in which blockchain may be applied. A good set of guidelines for when blockchain may usefully be integrated into a project has been given by [11]. Specifically, blockchain may be useful if the following conditions hold:

- Shared database - the project will require a database to be used as a shared ledger;

- Multiple writers - more than one entity will be generating transactions which will be modifying the database. Further, the writers will hold a copy of the database and relay transactions in a peer-to-peer fashion;

- Absence of trust - users are not willing to allow other users to modify the database entries which they own;

- Disintermediation - the multiple non-trusting entities want to operate without a centralized trusted intermediary;

- Transaction interaction - the transactions of different users depend on those of others;

- Set of rules - given the previous conditions, it is logical that the database should embed rules which restrict the transactions;

- Authoritative transaction $\log$ - the blockchain serves as the final transaction $\log$ on whose contents all users provably agree;

- Asset store - the blockchain serves as a ledger of real-world assets.

If all or most of these conditions hold, blockchain technology may well be applicable and useful for your project. Some reflection will show that many use cases in education have these conditions.

\section{Blockchain Platforms}

Several platforms for building blockchain systems have been developed in recent years. Amongst the most popular of these are the following:
- Ethereum - [12] is an open-source, public blockchain platform first proposed by Vitalik Buterin in 2013. Ethereum features Ether, a token (cryptocurrency) which can be used to pay for "gas", a unit of computation performed by smart contracts (scripts) which run on the Ethereum Virtual Machine (EVM). Ethereum supports a modified version of Nakamoto consensus (Bitcoin's consensus protocol). Ethereum is widely used for the development of Peer to Peer (P2P) Distributed (or Decentralized) Apps (Dapps).

- Hyperledger Fabric - [13] is a permissioned (private) blockchain infrastructure developed by IBM and Digital Asset which features a modular architecture, smart contracts, and configurable consensus and membership services. Besides being a private blockchain, Hyperledger Fabric differs from Ethereum in not having a built-in token, and in its degree of configurability, among other items. Hyperledger Fabric is one of a number of "Hyperledger" projects developed under the aegis of the Linux Foundation and is aimed at the enterprise with a special significance for Business to Business (B2B) applications.

- Corda - [14] was developed by the R3 blockchain technology company. Corda is an open-source distributed ledger and smart contract platform developed with the needs of business in mind. Like Hyperledger Fabric, Corda is permissioned and does not have a native token, however it is more specialized, aimed specifically at the needs of the financial services industry.

- Openchain - [15] developed by CoinPrism is an open-source blockchain platform for organizations that want to issue and manage digital assets in a secure, and scalable manner. Smart contracts are supported and partitioned consensus is used so that users can create unique instances that will have a single authority. Tokens on Openchain can be pegged to Bitcoin. Openchain uses a client-server architecture rather than the more widely used blockchain P2P architecture. There is also no miner required for consensus, so transactions are instant and free.

- BigChainDB - [16] is an open-source distributed storage system which aims to combine the advantages of NoSQL databases with blockchain (distributed storage, immutability, no central authority). BigChainDB is built out of number of NoSQL database nodes (e.g. MongoDB instances) which store immutable information about transactions which are kept synchronized by using the Tendermint Byzantine Fault Tolerance (BFT) scheme for consensus. BigChainDB can be either public, private, or permissioned.

\section{LITERATURE REVIEW}

In this section we will look at some recent representative projects in the application of blockchain technology in higher education.

A number of researchers have explored the use of blockchain to store university grades, i.e. university transcripts. A group at the University of Glasgow has developed a functional prototype for storage of student grades at the institution [17]. The platform chosen was 
Ethereum, hence it was built on a public blockchain. This was exploratory research, and they identified several challenges, including in the use of smart contracts to calculate grades in an algorithmic manner. Another project giving a prototype implementation is [3], where a private BigChainDB blockchain is used for storage of student transcripts (not grades within a course as in the previously described research, though). Initial results were promising. Yokubo [9] describes a prototype implementation of a university transcript system using an Ethereum private blockchain and ERC-20 tokens (a standard for tokens, which are needed to carry out smart contracts, on Ethereum) on that blockchain. Students are able to read their grades, while professors and administrative personnel can record grades.

EduCTX [18] is an ambitious project for the development of a higher education credit platform based on the concept of the European Credit Transfer and Accumulation System (ECTS), a framework which has been approved by the EU. The decentralized higher education credit and grading system can offer a globally unified viewpoint for students, higher education institutions, and other potential stakeholders such as prospective employers. A prototype implementation has been built on the ARK blockchain platform [19]. ARK is a public blockchain, but the researchers changed it to a private (permissioned) one by taking advantage of the flexible nature of ARK to change the parameters of the consensus algorithm used - DPoS (delegate proof of stake). Rules to ensure the validity of transactions on the blockchain have been defined. ECTX tokens are used to represents credits that students gain for completing courses (analogous to the way that ERC-20 tokens are used in the work above). The authors propose to apply the prototype system firstly at their home institution, the University of Maribor, and then at a select set of institutions of higher education. They anticipate that this or a similar system could potentially evolve into a unified, simplified and globally ubiquitous higher education credit and grading system.

A more theoretical investigation is given in [20] where the architecture for the Disciplina platform for student records is given and main issues arising from storing student records in a blockchain are analysed. Their platform incorporates both private blockchains (maintained by individual institutions of higher learning and public blockchains, managed by "Witnesses" who witness the fact that a private block was produced by a valid institution. A good discussion of the problems of privacy, provability, and data disclosure in this context are given in a theoretical manner.

Beyond traditional transcripts, blockchain is also being used or proposed for various alternative types of educational credentialing. Blockchain promises permanent authentication and storage for a myriad of alternative credentials made up of diverse microcredentials, nanodegrees, MOOCs, and certificates/badges from various types of training programmes. These credentials can then be directly controlled and managed by users [21].

Among the most well-known of these projects, MIT's Media Lab created blockcerts, a mobile app for educational credentialing built on Bitcoin [22]. At the Open University, researchers have developed the OpenLearn system built on the Ethereum public blockchain which awards OpenLearn badges for completing sections of a course and passing assessments [23]. The creators of that system are now working on a blockchain project to create a permanent distributed record of intellectual effort and associated reputational reward that instantiates and democratises educational reputation beyond the academic community [24]. Blockchain for Education [25] is another prototype system supporting the storage, retrieval and verification of certificates via blockchain technology. Certificates are an important means of proving lifelong learning achievement in today's environment, however they are susceptible to forgery. Blockchain helps to solve this problem. The prototype system uses Ethereum and its smart contracts to manage identities of registered certificate authorities and the hashes of certificates which are stored in a separate, centralized document management system, while the profile information of certificate authorities is stored using the Interplanetary File System (IPFS) distributed file system. Storing data off the blockchain allows it to be be deleted as is required, for example, by the European General Data Protection Regulation (GDPR) for personal information.

Other uses of blockchain in higher education have also been contemplated, including motivation, assessment, advising, etc. [26]. A prototype system for a blockchain based learning analytics platform built on Ethereum has been proposed as well [27].

\section{EXPERIMENTAL IMPLEMENTATION}

In this section we will describe an initial implementation of a prototype system for university transcripts which empower students using blockchain as well as NoSQL databases.

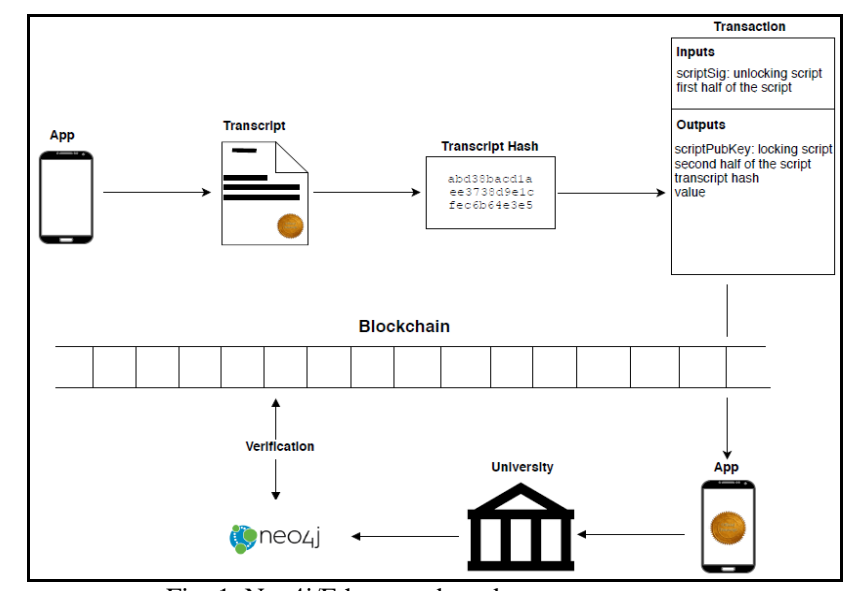

Fig. 1. Neo4j/Ethereum-based prototype system.

Ethereum, as noted previously, is a promising platform for implementation of this system since it is an open-source, public, blockchain-based distributed computing platform and operating system featuring smart contract (scripting) functionality. In our system, the university transcript data can be stored in blocks and functionality on the transcripts implemented via smart contracts. We have explored this possibility and enhanced it by hooking to the $\mathrm{Neo} 4 \mathrm{j}$ open-source NoSQL database. Neo4j is a native graph database since it implements the property graph model down to the storage level. A graph database fits well with the structure of the blockchain and can be used to interrogate and 
investigate parts of the blockchain using the cypher query language. The architecture of a prototype system using Ethereum and Neo4j is shown below.

An example query is: MATCH (a:tx), (b:block) RETURN $a, b$. The result of the query is a graph, as shown below in Fig. 2.

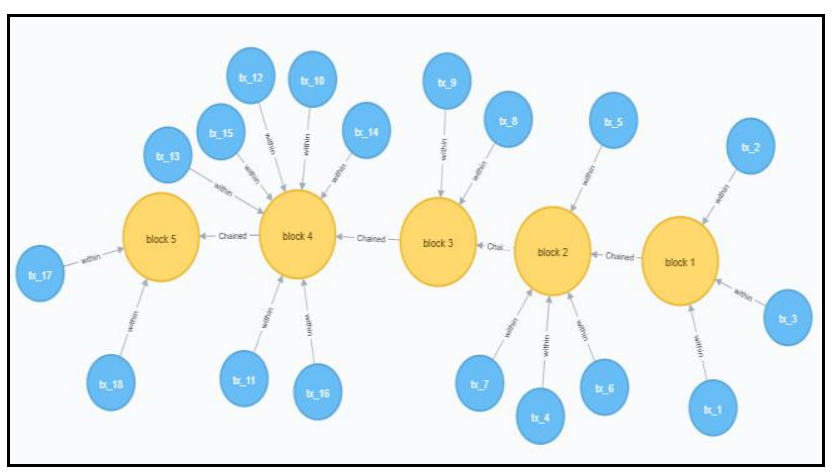

Fig. 2. Query results.

In the prototype, a small, relevant portion of the blockchain is imported into $\mathrm{Neo} 4 \mathrm{j}$ as shown below in Fig. 3 .

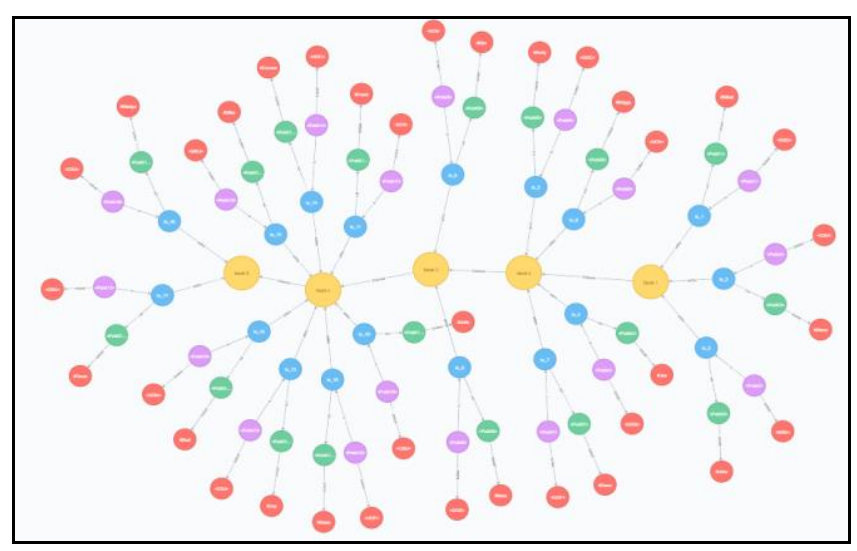

Fig. 2. Query results.

We can use Neo4j's cypher query language, e.g., to find nodes related to a student user Julie, with the following query, resulting in the results shown in Fig. 4. Query: MATCH $(a: t x),(b: b l o c k)<-[:$ within $]-(a: t x)$,

(c:output) $<-[:$ out $]-(a: t x)$,

$(\mathrm{d}:$ input $)-[$ : in $]->(\mathrm{a}:$ tx $),($ e:fromaddress )$<-[$ : locked $]-(d:$ input $)$,

(f:toaddress) <- [:locked] - (c:output) WHERE $\mathrm{b} . \mathrm{blockhash}=$ 'block 4' AND c.transcript $=$ '\#Julie' RETURN a,b, c, d,e, f

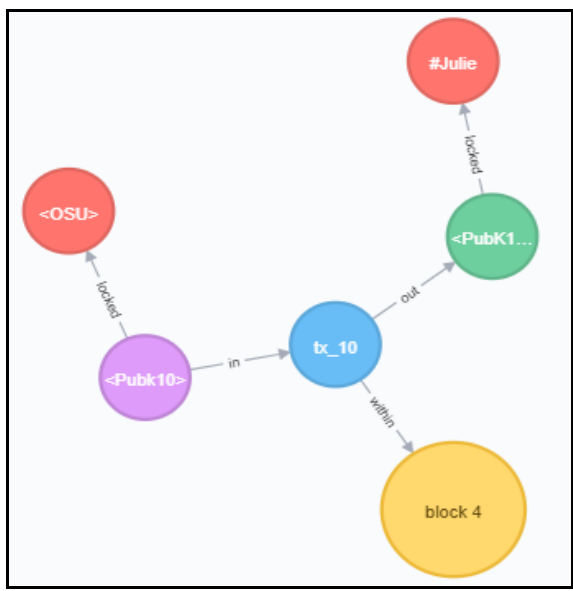

Fig. 4. Results of query.
Another approach we are exploring is the use of BigChainDB to securely store and retrieve student transcript records. BigChainDB is a hybrid model merging blockchain and distributed DB systems. The architecture of the prototype system using this approach is in Fig. 5 below.

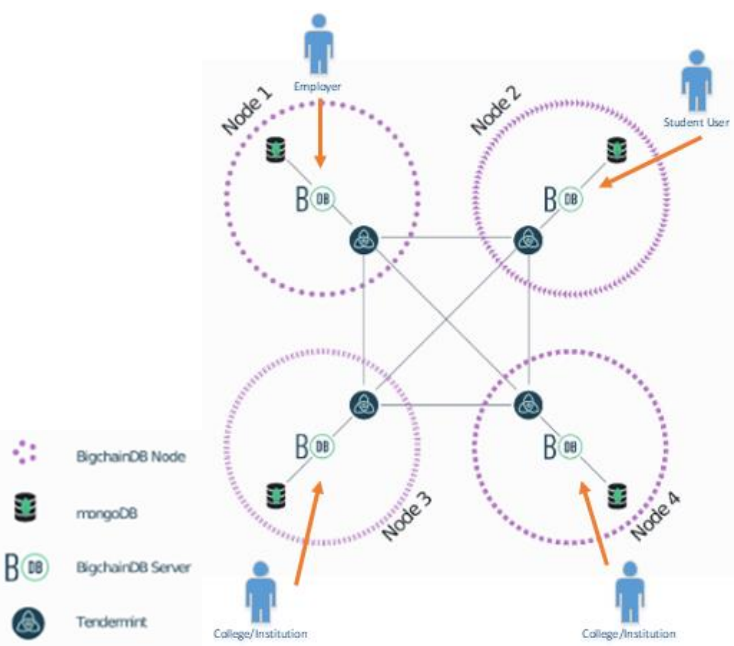

Fig. 5. Architecture of the BigChainDB-based prototype system.

A typical transcript process in this prototype system is shown in Fig. 6 below.

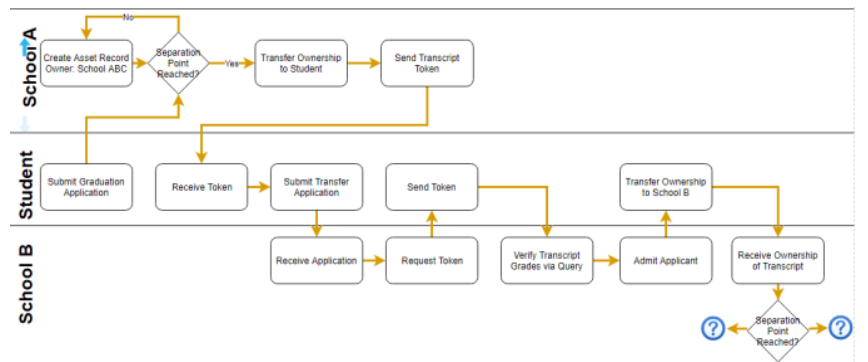

Fig. 6. Transcript process in the prototype system.

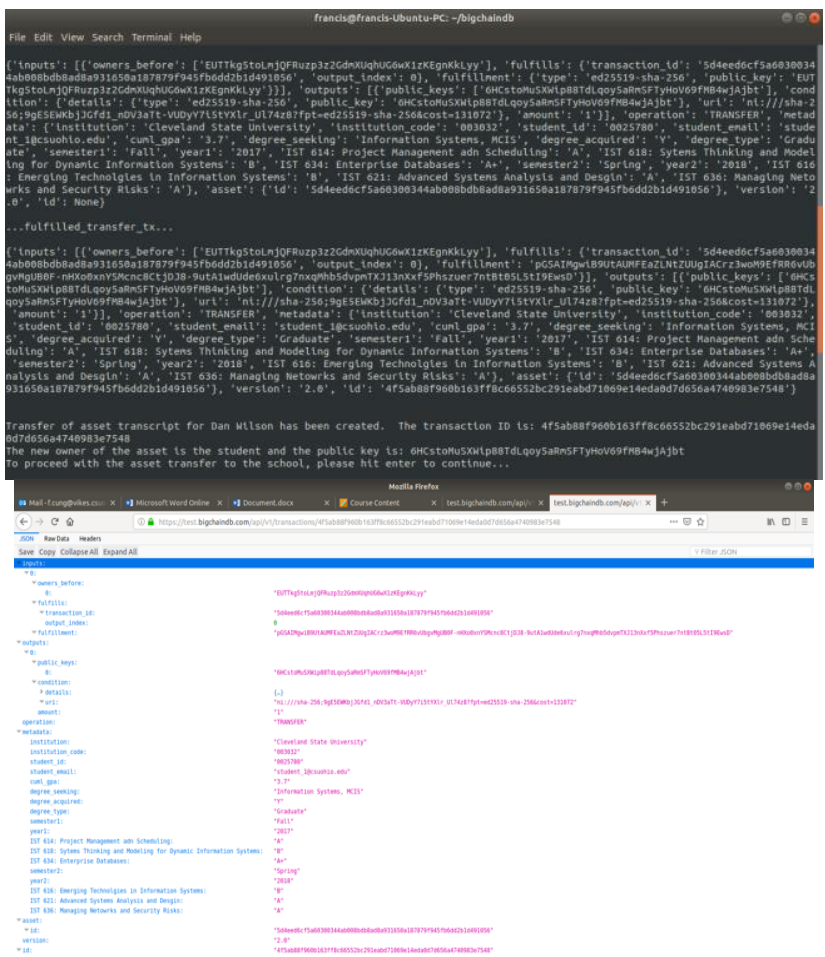

Fig. 7. Transfer of transcript and the transfer transaction block created. 
In this system, the transcript data is stored in BigChainDB's blockchain metadata. Transfer of transcript from a student to a university when the student enrolls at the university (so that the university may add data to the student's transcript) is shown below. The ownership of the transcript will be transferred back to the student when he separates from the university.

Initial results of the implementation are encouraging. We are able to store transcript data in the blockchain and manipulate it using NoSQL database functionality. Update and transfer of the transcript data is supported. We will be adding functionality to the systems and scaling them up with larger amounts of data in the near future. Evaluation and development of the two prototype systems is ongoing.

\section{CONCLUSION}

One of the factors influencing the choice of a particular blockchain platform is the availability of an API in a particular language. Researchers will naturally prefer a solution which allows them to program in their chosen language. Ethereum has proven to be a popular platform in higher education projects due to its smart contracts and generalist orientation, but other platforms have been widely used as well.

Among the areas in higher education where blockchain is currently being most widely used are a pair of emerging application areas which involve multiple, distributed actors, and which thus lend themselves naturally to the use of blockchain as a part of a system, namely for transfer of credits among highly mobile students crossing international borders (in the EU), and as a means of consolidating, new, diverse educational credentials (certificates, badges, MOOCs, etc.) in a more flexible, student-oriented approach to lifelong learning. Other areas of higher education could profit from the use of blockchain as well, however the criteria given in section 2 should be carefully considered before incorporating blockchain in order to avoid using inappropriate technology simply because it is new and "hot".

Our experimental results are currently being evaluated and extended in order to allow for use in a realistic scenario involving real university records.

\section{CONFLICT OF INTEREST}

The authors declare no conflict of interest.

\section{AUTHOR CONTRIBUTIONS}

Timothy Arndt supervised the development of the experimental implementations described in the paper by Cleveland State University students in the Master of Information Systems course IST 634 Enterprise Databases. The literature review, analysis, and writing of the paper were carried out jointly by both authors.

\section{ACKNOWLEDGMENT}

The help of Cleveland State University students in the Master of Information Systems course IST 634 Enterprise Databases is gratefully acknowledged.

\section{REFERENCES}

[1] S. Nakamoto. (2008). Bitcoin: A peer-to-peer electronic cash system. [Online]. Available: https://bitcoin.org/bitcoin.pdf

[2] M. Iansiti and K. R. Lakhani, "The truth about blockchain," Harvard Business Review, vol. 95, no. 1, pp. 118-127, 2017.

[3] T. Arndt, "Empowering university students with blockchain-based transcripts," in Proc. CELDA 2018, Budapest, Hungary, October 21-23, 2018.

[4] G. W. Peters and E. Panayi, "Understanding modern banking ledgers through blockchain technologies: Future of transaction processing and smart contracts on the internet of money," Banking beyond Banks and Money, Cham: Springer, 2016, pp. 239-278.

[5] S. Underwood, "Blockchain beyond bitcoin," Communications of the ACM, vol. 59, no. 11, pp. 15-17, 2016.

[6] F. Lamberti, V. Gatteschi, C. Demartini, C. Pranteda, and V. Santamaria, "Blockchain or not blockchain, that is the question of the insurance and other sectors," IT Professional, 2017.

[7] A. B. Ayed, "A conceptual secure blockchain-based electronic voting system," International Journal of Network Security \& Its Applications, vol. 93, 2017.

[8] H. Yumna et al., "Use of blockchain in education: A systematic literature review," Intelligent Information and Database Systems, ACIIDS 2019, Lecture Notes in Computer Science, vol. 11432, Cham: Springer, 2019, pp. 191-202.

[9] B. Yokubov, "Blockchain based storage of students career," Master degree thesis, Politecnico di Torino, 2018.

[10] Z. Zheng et al., "An overview of blockchain technology: Architecture, consensus, and future trends," IEEE $6^{\text {th }}$ International Conference on Big Data, pp. 557-564, 2017.

[11] G. Greenspan, "Avoiding the pointless blockchain project," 2015

[12] Ethereum. (2019). [Online]. Available: https://www.ethereum.org

[13] Hyperledger Fabric. (2019). [Online]. Available: https://www.hyperledger.org/projects/fabric

[14] Corda. (2019). [Online]. Available: https://www.r3.com/corda-platform/

[15] Openchain. (2019). [Online]. Available: https://www.openchain.org

[16] BigChainDB. (2019). [Online]. Available: https://www.bigchaindb.com

[17] J. Rooksby and K. Dimitrov, "Trustless education? A blockchain system for university grades," New Value Transactions Understanding and Designing for Distributed Autonomous Organisations Workshop at DIS 2017, 2017.

[18] M. Turkanović et al., "EduCTX: A blockchain-based higher education credit platform," IEEE Access, vol. 6, pp. 5112-5127, 2018.

[19] ARK. (2019). [Online]. Available: https://ark.io

[20] K. Kuvshinov. (2018). Disciplina: Blockchain for education. [Online]. Available:

https://www.semanticscholar.org/paper/Disciplina-\%3A-Blockchain-f or-Education-Kuvshinov-Nikiforov/958c1a5760b3f41f65482593eb8e $365 \mathrm{dfc} 4 \mathrm{ccf} 3 \mathrm{f}$

[21] M. Jirgensons and J, Kapenicks, "Blockchain and the future of digital learning credential assessment and management," Journal of Teacher Education for Sustainability, vol. 20, pp. 145-156, 2018.

[22] Blockcerts. (2019). The Open Standard for Blockchain Credentials. [Online]. Available: https://www.blockcerts.org

[23] OpenLearn. (2019). [Online].

Available: https://www.open.edu/openlearn/get-started/badges-come-openlearn

[24] M. Sharples and J. Domingue, "The blockchain and kudos: A distributed system for educational record, reputation and reward," Adaptive and Adaptable Learning, EC-TEL 2016, Lecture Notes in Computer Science, vol. 9891, Cham: Springer, 2016, pp. 490-496.

[25] W. Gräther et al., "Blockchain for education: Lifelong learning passport," in Proc. the $1^{\text {st }}$ ERCIM Blockchain Workshop 2018, Reports of the Society for Socially Embedded Technologies, 2018.

[26] G. Chen et al., "Exploring blockchain technology and its potential applications for education," Smart Learning Environments, vol. 5, no. $1,2018$.

[27] B. Ocheja et al., "Connecting decentralized learning records: A blockchain based learning analytics platform," in Proc. International Conference on Learning Analytics and Knowledge, Sydney, Australia, March 2018

Copyright (C) 2020 by the authors. This is an open access article distributed under the Creative Commons Attribution License which permits unrestricted use, distribution, and reproduction in any medium, provided the original work is properly cited (CC BY 4.0). 


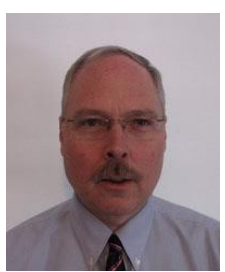

Timothy Arndt received the Ph.D. in computer science from the University of Pittsburgh, Pittsburgh, PA USA in 1991, the master of science in computer and information science from the University of Florida, Gainesville, FL, USA in 1985 and the bachelor of science degree in computer science and mathematics from Purdue University, West Lafayette, IN, USA in 1984

$\mathrm{He}$ is a professor in the Department of Information Systems and the Department of Electrical Engineering and Computer Science, Cleveland State University, Cleveland OH, USA. He has previously been an assistant professor at Texas State University and Lamar University, an Adjunct Faculty member at the Universita' del Sannio, and a consultant with Italdata S.p.A..

Dr. Arndt is a senior member of the Association for Computing Machinery. He was the co-chair of the distributed multimedia systems 2005 conference and has been a guest editor for special issues of the Journal of Visual Language and Computing and the International Journal of Software Engineering and Knowledge Engineering. He is currently an editor of the Journal of Visual Languages and Computing and a member of the Editorial Review Boards of the Journal of Distance Education Technology and the Journal of Software.

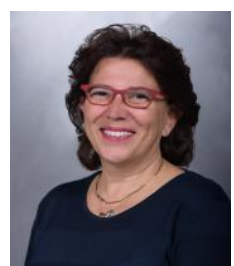

Angela Guercio received the Ph.D. in computer science from Kent State University, Kent, OH, USA, in 2004, the master of science in computer and information sciences from the Knowledge Systems Institute, Skokie, IL, USA, in 2000, and the Laurea degree in computer science "cum laude" from the University of Salerno, Salerno, Italy, in 1984.

She is an associate professor of computer science in the Department of Computer Science at Kent State University and resides at the Stark campus. She has previously been an assistant professor at Hiram College, Hiram, Ohio, an adjunct research visiting professor at Lamar University, Beaumont, Texas, and a senior research associate at the University of Salerno, Lancusi, Italy.

Dr. Guercio is a member of the IEEE, the Association for Computing Machinery, and the IEEE Computer Society. She was conference Chair of the Distributed Multimedia Systems 2016 and co-Chair in 2015 and 2005, co-Chair of the International Workshop on Distance Education Technologies 2013, and of multiple editions of the Ohio Celebration of Women in Computing. She has been a guest editor for special issues of the Journal of Visual Languages and Computing and the International Journal of Software Engineering and Knowledge Engineering. She is currently a member of the Editorial Board of the Journal of Visual Language and Computing and of the International Journal on Advances in Network and Services. 